\title{
Análise biométrica em frutos e sensorial do suco de Guabiju (Myrcianthes pungens)
}

\section{Biometric analysis in fruit and and sensorial of Guabiju (Myrcianthes pungens)}

\author{
Marlene Aparecida Rodrigues ${ }^{(1}{ }^{1}$, Divanilde Guerra ${ }^{\left(\mathbb{D}^{2}\right.}$, Robson Evaldo Gehlen Bohrer ${ }^{(\mathrm{D})}$, Danni Maisa Da \\ Silva $\mathbb{1}^{2}$, Fernanda Hart Weber ${ }^{2}{ }^{2}$, Marlon de Castro Vasconcelos $\left(^{2}\right.$ \\ ${ }^{1}$ Bacharel em Gestão Ambiental. Universidade Estadual do Rio Grande do Sul, Unidade em Três Passos (RS), + 55 55 81109742 , \\ marlene.aparecida.rodrigues@gmail.com. ${ }^{2}$ Professores Doutores da Universidade Estadual do Rio Grande do Sul, Unidade em Três Passos (RS), divanilde- \\ guerra@uergs.edu.br; robson-bohrer@uergs.edu.br; danni-silva@uergs.edu.br; fernanda-hart@uergs.edu.br; marlon-vasconcelos@uergs.edu.br.
}

$\mathrm{NOT} \mathrm{A}$

Recebido: 09/10/2020

Aprovado: 26/12/2020

Palavras-chave:

Mirtáceas

Guabijuzeiro

Aceitação do suco

Key words:

Mirtacea

Guabijuzeiro

Juice acceptance

\section{R E S U M O}

O guabijuzeiro (Myrcianthes pungens) se destaca pela abrangência em todo o território nacional, pela produção e sabor adocicado dos frutos, mas poucos estudos foram conduzidos com esta espécie até o momento. $\mathrm{O}$ objetivo do presente trabalho foi realizar a caracterização biométrica dos frutos e análise sensorial do suco produzido de três acessos de guabiju. A metodologia utilizada consistiu na coleta dos frutos para análises de massa de frutos, rendimento de polpa, sólidos solúveis totais e análise sensorial do suco sendo realizado teste de aceitação com 35 avaliadores não treinados. Como resultados observou-se que a média da massa de frutos foi de 3,57 $\mathrm{g}$, a massa de polpa foi de $1,81 \mathrm{~g}$, o rendimento de polpa foi de $49,67 \%$ e os sólidos solúveis totais foi de $14,27 \%$. Com relação a produção de suco, o acesso 1 produziu $300 \mathrm{~mL}$, o acesso $2200 \mathrm{~mL}$ e o acesso $3400 \mathrm{~mL}$. Com relação a análise sensorial, o acesso 1 obteve a melhor nota de aceitação por 11,42\% dos avaliadores, o acesso 2 por $80 \%$ dos avaliadores e o acesso 3 por 8,57 \% dos avaliadores, o que mostra o potencial de utilização destes frutos para o desenvolvimento de sucos com frutos exóticos. Pode-se concluir que existe variação entre os acessos na biometria de frutos, produção de suco e grau de aceitação dos provadores do suco de guabiju.

\section{A B S T R A C T}

The guabijuzeiro (Myrcianthes pungens) stands out for its coverage throughout the national territory and for the production and sweet taste of the fruits, but few studies have been conducted with this species so far. The objective of the present work was to carry out the biometric characterization of the fruits and the sensory analysis of the juice produced from three guabiju accessions. The methodology used consisted of collecting the fruits for analysis of fruit mass, pulp mass, pulp yield and Brix grade and for the production of guabiju juice. An acceptance test was carried out with 35 untrained evaluators. As a result, it was observed that the average fruit mass was $3.57 \mathrm{~g}$, the pulp mass was $1.81 \mathrm{~g}$, the pulp yield was $49.67 \%$ and the average Brix grade was $14.27 \%$. Regarding juice production, access 1 produced $300 \mathrm{~mL}$, access $2200 \mathrm{~mL}$ and access $3400 \mathrm{~mL}$. Regarding sensory analysis, access 1 obtained the best acceptance score by $11.42 \%$ of the evaluators, access 2 by $80 \%$ of the evaluators and access 3 by $8.57 \%$ of the evaluators, which shows the potential of use of these fruits for the development of juices with exotic fruits. It can be concluded that there is variation between accessions in fruit biometry, juice production and degree of acceptance by guabiju juice tasters.

\section{INTRODUÇÃO}

No Brasil, a produção de frutas em larga escala é essencialmente realizada com espécies exóticas, contudo o país apresenta grande diversidade vegetal, possuindo espécies frutíferas nativas, que ainda são pouco exploradas e até mesmo desconhecidas, mas que apresentam grande potencial e valor econômico. Dentre elas, destacam-se principalmente as da família Myrtaceae, como a pitangueira (Eugenia uniflora L.), cerejeira-do-mato (Eugenia involucrata DC.), uvalheira (Eugenia pyriformis Cambess.), guabirobeira (Campomanesia xantocarpa O. Berg), araçazeiro (Psidium cattleyanum Sabine)

$\begin{array}{lc}\text { Revista Verde } & \text { v. } 16, \text { n.2, abr.-jun, p.111-116, } 2021 \\ \text { ISSN 1981-8203 } & \text { doi: } 10.18378 / \text { rvads.v16i2.8495 } \\ \text { Pombal, Paraíba, Brasil } & \end{array}$


e guabijuzeiro (Myrcianthes pungens Legrand) (SERAGLIO et al., 2018). Os frutos podem ser explorados comercialmente, visando à diversificação da produção e do consumo in natura ou podem ser transformados em subprodutos pela agroindústria alimentícia (DANNER et al., 2010). Ainda, apresentam propriedades farmacêuticas e nutracêuticas, pela presença de compostos secundários fitoterápicos, aromáticos e antioxidantes (CARVALHO et al., 2014).

O guabijuzeiro apresenta ocorrência natural na Argentina, Bolívia, Paraguai, Uruguai e Brasil, onde pode ser encontrado desde São Paulo até o Rio Grande do Sul, sendo eventual em todas as formações florestais (LORENZI, LACERDA, 2006). Seus frutos são globosos, com polpa suculenta e adocicada, podendo ser cultivado para a comercialização e alimentação humana, tanto para consumo in natura quanto para o processamento na forma de doces e geleias (SOBUCKI et al., 2015; SOUZA et al., 2018). Não obstante, os frutos apresentam alto conteúdo de polifenóis totais, flavonoides e antocianinas, além dos extratos apresentarem alta capacidade antioxidante quando comparada com a de outros micronutrientes, como a vitamina E (SOUZA et al., 2018).

Embora sejam de grande importância, muitas espécies frutíferas da família Mirtácea como o guabiju, apresentam escassez ou mesmo ausência de dados relativos à sua morfologia, produção, características fisiológicas e fenologia, as quais são importantes para a descrição e caracterização dos diversos genótipos existentes. Esses dados servem de base para a incorporação de muitas espécies aos sistemas produtivos comerciais, contribuindo também para a conservação dos recursos genéticos e difusão de seu consumo (CARVALHO et al., 2003). Nesse sentido, a biometria constitui importante instrumento para detectar a variabilidade genética dentro de populações de uma mesma espécie, as relações entre esta variabilidade e os fatores ambientais, bem como para a identificação de genótipos mais promissores na produção de frutos com qualidade morfológica e nutricional (CARVALHO et al., 2003).

Conforme Braga et al. (2020), o aumento do consumo de frutas in natura e sucos naturais é uma tendência mundial que pode ser aproveitada pelo Brasil como forma de incentivar o aumento da produção e da qualidade das frutas, bem como, a geração de emprego e renda. Segundo Silva et al. (2011), frutas nativas e exóticas e seus derivados, estão com seu consumo em ascensão por serem consideradas um alimento saudável, balanceado, funcional e diversificado, bem como, para agregar atributos de qualidade diferencial ao produto (MOURA et al., 2016).

Muitas espécies frutíferas nativas, como o guabijuzeiro, têm consumo restrito aos locais de produção e no pico da safra devido à perecibilidade. Porém uma forma de disponibiliza-las por mais tempo e a maiores distâncias é através do processamento, ou seja, a transformação da matéria-prima perecível em armazenável e comercializável, visto que muitas destas apresentam qualidade sensorial, o que é fundamental para seu aproveitamento tecnológico (BOESSO et al., 2006).

$\mathrm{O}$ guabijuzeiro se destaca pela sua abrangência em todo o território nacional, porém poucos estudos foram conduzidos com esta espécie até o momento e inexistem trabalhos avaliando a produção e aceitação do suco, sendo, portanto, este o primeiro a avalia-lo. Desta forma, objetivou-se caracterizar biometricamente os frutos e realizar a análise sensorial do suco de guabiju oriundos de três acessos diferentes.

\section{MATERIAL E MÉTODOS}

A pesquisa foi realizada no município de Três Passos que se localiza a uma latitude $27^{\circ} 27^{\prime}$ sul e longitude $53^{\circ} 55^{\prime}$ 'Oeste na região Noroeste do estado do Rio Grande do Sul, inserido no bioma Mata Atlântica (IBGE, 2020).

\section{Caracterização biométrica dos frutos}

Acessos de guabijuzeiros foram monitorados no município de Três Passos por apresentarem frutos grandes e de sabor adocicado. Em fevereiro de 2018, quando estes apresentavam frutos em fase de maturação, procedeu-se a colheita e avalição das características biométricas no laboratório da Universidade Estadual do Rio Grande do Sul (UERGS), unidade em Trêss Passos/RS.

Dos acessos monitorados foram escolhidos três, acesso 1 (coordenadas S $27^{\circ} 27^{\prime} 31.2^{\prime \prime}$ e W $053^{\circ} 56^{\prime} 13.5^{\prime \prime}$ ), acesso 2 (coordenadas S $27^{\circ} 27^{\prime} 43.6^{\prime \prime}$ e W $053^{\circ} 55^{\prime} 56.1^{\prime \prime}$ ) e acesso 3 (coordenadas S $27^{\circ} 27^{\prime} 28.6^{\prime \prime}$ e W $053^{\circ} 56^{\prime} 16.2^{\prime \prime}$ ). Destes foram coletados 10 frutos de cada, os quais estavam distribuídos em todos os quadrantes da planta. Estes foram avaliados quanto a produção de massa com auxílio de uma balança de precisão (Adventurer Analitica). Após ocorreu a separação de forma individual da polpa para a determinação da massa de polpa (RODRIGUES et al., 2020). A determinação do rendimento de polpa foi realizada pela divisão dos valores de massa fresca de polpa pelo valor obtido de massa de fruto inteiro, e o teor de sólidos solúveis totais - ${ }^{\circ}$ Brix $(\%)$ em cada acesso foi medido com a utilização de um refratômetro portátil (Hand Held Refractrometer 32/ATC) utilizando metodologias já descritas por Sobucki et al. (2015) em diversas Mirtáceas.

\section{Análise sensorial do suco}

Foram coletados $1000 \mathrm{~g}$ de frutos de cada um dos acessos e estes foram submetidos ao processo de pré-seleção sendo retirados os galhos, folhas, pedúnculos, frutos danificados e em fase de senescência avançada, segundo metodologia de Boesso et al. (2015). Os frutos classificados para a extração do suco foram lavados em água corrente e sanitizados em solução de hipoclorito de sódio a $2 \%$.

A metodologia empregada para obtenção do suco foi a extração por arraste de vapor utilizando extratora de suco artesanal (Suga Sucos), onde a água em ebulição fica na parte inferior do equipamento e o vapor d'água sobe extraindo o suco dos frutos, que ficam suspensos em uma peneira. Os mesmos permaneceram em contato com o vapor durante 60 minutos a 80 ${ }^{\circ} \mathrm{C}$, sendo o suco coletado nesta mesma temperatura e envasado em garrafas de vidro âmbar de $600 \mathrm{~mL}$, vedadas com tampa metálica em recravadora. As amostras foram armazenadas em temperatura ambiente e ao abrigo da luz. $\mathrm{O}$ processo de extração utilizado é uma derivação do método de Welch, descrito por Guerra (2016).

Para a realização da análise sensorial procedeu-se a diluição dos sucos integrais de Guabiju na proporção 1:2 (água: suco). Foram oferecidos $25 \mathrm{~mL}$ de amostra de suco, por acesso 
de guabiju para uma equipe avaliadora de 35 provadores adultos, funcionários, alunos e professores da Universidade, todos voluntários e sem treinamento. Os avaliadores foram esclarecidos com relação ao objetivo do trabalho e ao preenchimento da ficha de avaliação. Foram disponibilizados copos com água e bolacha água e sal entre as degustações de uma amostra e outra, para que ocorresse a limpeza das papilas gustativas, não havendo interferência de gosto entre a avaliação das amostras. Utilizou-se o Teste Triangular (BARROSO, 2013), que foi conduzido de maneira que os provadores pudessem escolher a amostra que mais gostaram, levando em consideração os atributos de aparência, aroma, sabor e textura.

Os dados foram analisados por meio da ANOVA de um fator seguida de teste de Tukey e quando não foi possível a homogeneidade de variâncias e normalidade de resíduos, utilizou-se o teste de Kruskal-Wallis. Todas as análises foram realizadas no ambiente estatístico $\mathrm{R}$ v 3.6 ( $\mathrm{R}$ CORE TEAM, 2020).

\section{RESULTADOS E DISCUSSÃO}

A análise biométrica dos frutos revelou a existência de variabilidade entre os acessos avaliados. A média da massa dos frutos inteiros foi de $3,57 \mathrm{~g}$ (Tabela 1). Estes valores são similares aos obtidos por Reis et al. (2016) que identificaram uma média de 3,41 $\mathrm{g}$ em acessos de guabiju, porém diferem dos resultados obtidos por Dias et al. (2011), em pitangueiras, espécie também da família das mirtáceas, que observaram a massa de frutos variando entre 1,28 a 6,52 g.

Tabela 1. Análise biométrica dos frutos de guabiju (Myrcianthes pungens), Três Passos - Rio Grande do Sul.

\begin{tabular}{lllll}
\hline Acessos & $\begin{array}{l}\text { Massa } \\
\text { Frutos } \\
(\mathrm{g})\end{array}$ & $\begin{array}{l}\text { Massa } \\
\text { Polpa } \\
(\mathrm{g})\end{array}$ & $\begin{array}{l}\text { Rendimento } \\
\text { Polpa } \\
(\%)\end{array}$ & $\begin{array}{l}\text { Sólidos } \\
\text { Solúveis } \\
\text { Totais }(\%)\end{array}$ \\
\hline 1 & $3,04 \mathrm{~b}$ & $1,48 \mathrm{~b}$ & $48,68 \mathrm{~b}$ & $13,50 \mathrm{~b}$ \\
2 & $3,10 \mathrm{~b}$ & $1,32 \mathrm{~b}$ & $42,58 \mathrm{c}$ & $13,50 \mathrm{~b}$ \\
3 & $4,57 \mathrm{a}$ & $2,64 \mathrm{a}$ & $57,76 \mathrm{a}$ & $15,80 \mathrm{a}$ \\
\hline Média & 3,57 & 1,81 & 49,67 & 14,27 \\
DP & 0,87 & 0,72 & 7,64 & 1,33 \\
CV \% & 24,27 & 39,72 & 15,37 & 9,30 \\
\hline
\end{tabular}

Médias seguidas por uma mesma letra, em cada coluna, não diferem estatisticamente (Tukey, $\mathrm{p}>0,05$ ).

Diferenças significativas foram observadas entre os acessos, com variação no tamanho dos frutos de 3,04 a 4,57 g. A superioridade de massa de frutos do acesso 3 (4,57 g), pode estar associada às condições ambientais, de manejo e constituição genética das plantas. Esta afirmação estaria de acordo ao descrito por Carvalho et al. (2003), de que na maioria das espécies, grandes variações são observadas no tamanho e massa dos frutos devido à origem diferenciada das plantas-mãe, bem como, a segregação causada pela alogamia, comum nas fruteiras nativas da família das Mirtáceas. Neste sentido, a determinação do peso médio de frutos é uma característica importante para o mercado de frutas frescas, uma vez que aqueles mais pesados são também os de maior tamanho, tornando-se mais atrativos para os consumidores. Ainda, o peso médio é uma característica importante, pois os maiores frutos são os mais valorizados para a industrialização por apresentarem mais polpa e por consequência mais suco (DIAS et al., 2011). Portanto, a caracterização do tamanho dos frutos é muito importante, visto que este parâmetro apresenta relação direta com a produção de polpa e suco.

As diferenças significativas entre os acessos podem também estar associadas às condições climáticas, as quais alteraram diversas características em guabijuzeiro, como as biométricas e as reprodutivas, sendo estas, possíveis fatores para as diferenças neste estudo. Esta hipótese estaria de acordo ao observado por Veit et al. (2019) que avaliaram o ciclo reprodutivo do guabijuzeiro nas regiões noroeste, nordeste e metropolitana do estado do Rio Grande do Sul e observaram variação na fenologia do ciclo reprodutivo com duração de 95 , 147 dias e 133 dias, respectivamente, além de diferenças em características dos frutos e atribuíram estas diferenças as condições ambientais diferenciadas entre os locais de coleta de frutos.

Na avaliação da massa de polpa observou-se a média de $1,81 \mathrm{~g}$, com variação de 1,32 a 2,64 g (Tabela 1), sendo possível identificar a superioridade do acesso 3 na produção de polpa, com 2,64 g. Desta forma, é possível inferir a existência de correlação positiva entre as características avaliadas, ou seja, quanto maior o tamanho do fruto, maior a quantidade de polpa, visto que o acesso 3 apresentou superioridade nestes dois parâmetros avaliados. Corroborando com Dias et al. (2011) que descrevem a correlação positiva entre os parametros tamanho de fruto e massa de polpa, bem como, estes autores destacam que quanto maior os valores de polpa dos frutos, melhor, tanto para o consumo in natura quanto para a indústria. Diante disso, pode-se afirmar que para fins de produção de alimentos processados (sucos, licores, sorvertes, etc.) o acesso 3 resultaria em maior produção, permitindo, portanto, sua indicação para fins industriais, bem como para consumo in natura.

Com relação ao rendimento de polpa, observou-se a média de 49,67 \% (Tabela 1), com variação de 42,58 \% (acesso 2) a $57,76 \%$ (acesso 3). A percentagem de produção de polpa, assim como a massa de polpa, são características muito importantes em processos de industrialização dos frutos, bem como, quando o destino dos frutos é para o consumo in natura, a porcentagem de polpa é a característica mais importante, uma vez que a casca e a(s) semente(s) são descartadas (ZERBIELLI et al., 2016).

Devido à importância da percentagem de polpa em espécies frutíferas, fez-se o comparativo dos resultados deste estudo a outros e observou-se a inferioridade da produção de polpa nos acessos de guabijuzeiros avaliados, visto que Dias et al. (2011) obtiveram o percentual de polpa com amplitude de 68,23 a $88,13 \%$ e média de $79,46 \%$; Bezerra et al. (2004), obtiveram $80 \%$; e Borges et al. (2010) obtiveram média de 59,1 $\%$ em frutos de 20 matrizes na Reserva Ecológica do Clube Caça e Pesca Itororó no município de Uberlândia. Porém, apesar da menor produção de polpa observada neste estudo, a qual pode estar associada ao número de sementes e ao tamanho destas, os frutos apresentam grande potencial alimentar, pois são ricos em polifenóis, flavonoides, antocianinas e compostos fenólicos (ANDRADE et al., 2011; SOUZA et al., 2018), o que pode valorizar a exploração comercial destes. Ainda, conforme Borges et al. (2010), o baixo rendimento percentual de polpa 
não se constitui em característica que inviabilize a utilização de uma determinada espécie, seja como fruta fresca ou para aproveitamento industrial, isso devido à grande aceitação que a mesma pode ter, contudo seu volume de suco é reduzido em detrimento ao volume de sementes.

Para a percentagem de sólidos solúveis totais, a média foi de $14,27 \%$, com variação de 13,5 a $15,8 \%$ (Tabela 1 ), sendo que o acesso 3 apresentou o maior valor (15,80\%), diferindo estatisticamente dos demais acessos. Ao compararmos os resultados obtidos no presente trabalho (amplitude de 13,5 a $15,8 \%$ ) aos trabalhos de Dias et al. (2011) em pitangas que observou variação de 9 a $15,30 \%$ e ao de Alvarenga et al. (2014) em uvaia que obteve $8,58 \%$ de sólidos solúveis, pode-se inferir que os frutos avaliados neste estudo apresentam teor de sólidos solúveis totais mais alto que as pitangas, os guabijus e as uvaias colhidas em outras regiões, sendo, portanto, melhores para o consumo in natura e mais atrativos para a indústria na forma de processados. Esta afirmação corrobora ao proposto por Dias et al. (2011) de que os frutos com altos teores de sólidos solúveis totais são preferidos, tanto para o consumo in natura quanto para a industrialização, por propiciar maior rendimento no processamento, em razão da maior quantidade de néctar produzido por quantidade de polpa. Bem como, elevados teores destes constituintes na matéria prima implicam em menor adição de açúcares, menor tempo de evaporação da água, menor gasto de energia e maior rendimento do produto, resultando em maior economia no processamento de alimentos (REIS et al., 2016)

Os teores de sólidos solúveis podem variar entre espécies, cultivares, grau de maturação, clima e condições de cultivo, como luminosidade e temperatura (ZERBIELLI et al., 2016). Portanto, os resultados obtidos neste trabalho podem estar associados a fatores climáticos, o que estaria em acordo ao observado por Reis et al. (2016) que avaliando acessos de guabiju coletados em Caxias do Sul - Rio Grande do Sul, obtiveram média de 9,67 \% de sólidos solúveis totais, valores inferiores aos obtidos neste estudo, o que pode reforçar a afirmação de que as condições ambientais exerçam influência sobre esta característica. Afirmação esta que corrobora com Alvarenga et al. (2014) de que o tipo de fruto, cultivar, condições edafoclimáticas e época de colheita interferem nos teores de sólidos solúveis.

Na determinação da quantidade de suco, observou-se que o acesso 1 produziu $300 \mathrm{~mL}$ de suco, o acesso 2, $200 \mathrm{~mL}$ e o acesso $3400 \mathrm{~mL}$. Estes resultados devem estar associados às características biométricas dos frutos, visto que diferenças significativas foram observadas entre os acessos (Tabela 1). Na avaliação sensorial, a qual permitiu identificar que o suco do acesso 1 obteve a aceitação de 4 provadores $(11,42 \%)$, do acesso 2 de 28 provadores $(80 \%)$ e do acesso 3 de 3 provadores $(8,57 \%)$. Desta forma, o suco do acesso 2 estaria apto a ser processado e em condições de ser comercializado devido a boa aceitação. Pois no processo de desenvolvimento de novos produtos a determinação da aceitação e/ou preferência do produto se torna indispensável (BRAGA et al., 2020). Ainda, de acordo com Gularte (2009), para que um produto seja considerado como aceito, em termos de suas características sensoriais de qualidade em uma percepção global é necessário que obtenha um IA (índice de aceitabilidade) de no mínimo 70
$\%$, valores estes que foram superados neste estudo com o suco do acesso 2.

A maior aceitação do suco do acesso 2 pode estar relacionada às características biométricas, como a massa e percentagem de polpa, visto que neste, observou-se os menores valores para estes parâmetros com 1,32 g de massa de polpa e $42,58 \%$ de rendimento de polpa (Tabela 1). Esta informação permite inferir que os frutos com menos polpa apresentem maior concentração de sólidos solúveis totais por grama de suco produzido, ou seja, menos suco no processo de extração, porém com maior concentração de sólidos solúveis totais e melhor aceitação dos provadores.

Os frutos dos três acessos avaliados apresentaram teor de sólidos solúveis totais elevado (Tabela 1), quando comparado a pitangas (DIAS et al., 2011) e uvaias (ALVARENGA et al., 2014), com destaque para o acesso 3 que atingiu valores estatisticamente superiores aos demais. Neste contexto, o fato dos sólidos solúveis totais do acesso 2, o mais aceito pelos provadores, não ser o mais elevado, justifica-se pela constituição do SST, o qual é composto por outros componentes, que não apenas açúcares. De acordo com Braga et al. (2020), os sólidos solúveis são constituídos por substâncias, como: açúcares, ácidos, vitamina C e algumas pectinas, os quais são usados como índice dos açúcares totais em frutos. Além de indicarem o grau de maturidade através do seu ${ }^{\circ}$ Brix (VERGANA et al., 2020).

Frutas com altos teores de sólidos solúveis totais são desejáveis para o consumo in natura, e para a indústria, pois nas avaliações por provadores são as que, em geral, aparecem com notas mais altas nos atributos avaliados. A maior concentração de açúcares e outros componentes permite a redução deste no processamento, embora, no Brasil, a indústria alimentícia, em geral, utilize um teor de 20 a $30 \%$ de açúcar mais alto em suas formulações, se comparada às indústrias europeias e asiáticas (COHEN et al., 2010). Portanto, o uso excessivo de açúcar acaba por influenciar o paladar dos provadores, afirmação esta que corrobora ao estudo de Bach et al. (2019) onde foram avaliados sensorialmente sucos de laranja, reconstituídos e adoçados com mel, açúcar mascavo e açúcar refinado. O suco de laranja adoçado com açúcar refinado apresentou-se como a melhor opção para o público de provadores, seguido do suco de laranja com mel e o suco de laranja com açúcar mascavo.

A procura por alimentos com qualidade, praticidade, propriedades funcionais, segurança e preços acessíveis vem crescendo e, assim, as inovações na elaboração dos produtos é objetivo constante na indústria de alimentos, que visa agregar atributos de qualidade diferencial ao produto (MOURA et al., 2016). Portanto, com base nos resultados obtidos neste estudo, o consumo in natura dos frutos de guabiju ou na forma de suco podem resultar em inúmeros benefícios aos consumidores, visto que estes apresentam alto conteúdo de polifenóis totais, flavonoides e antocianinas, além de compostos fenólicos que apresentam alta capacidade antioxidante (ANDRADE et al., 2011; SOUZA et al., 2018).

\section{CONCLUSÕES}

Os frutos de Guabiju do acesso 3 apresentam diferenças biométricas para massa de frutos, massa de polpa, rendimento 
de polpa e sólidos solúveis totais na comparação com os demais acessos.

Os acessos apresentam diferenças no rendimento de suco, sendo que a maior aceitação na análise sensorial dos provadores ocorreu com o suco do acesso 2.

\section{REFERÊNCIAS}

ALVARENGA, C. A. G.; CIABOTTI, S; PASSAGLIA, K. A.; JESUS, E. J. Avaliação da fruta de Eugenia uvalha cambes sob estádios de desenvolvimento. Global Science Technology, $\quad 7: \quad 67-75, \quad 2014 . \quad \underline{10.14688 / 1984-}$ $\underline{3801 / g s t . v 7 n 1 p 67-75}$

ANDRADE, J. M. M.; ABOY, A. L.; APEL, M. A.; RASEIRA, M. C. B.; PEREIRA, J. F. M.; HENRIQUES, A. T. Phenolic Composition in different genotypes of guabiju fruits. Joournal of Food Science, 76: 1181-1187, 2011. 10.1111/j.1750$\underline{\text { 3841.2011.02375.x }}$

BACK, P. I. K.; STEINHAUS, J.; RAMIRES, M. F; STOLL, D; VASCONCELOS, M. C; WEBER, F. H. Sensorial de suco de laranja com diferentes açúcares. Revista Verde de Agroecologia, 14: 480-484. 2019. 10.18378/rvads.v14i3.6317

BARROSO, C.M. Proposta de Análise Estatística de Testes Triangulares Considerando a Distribuição Binominal. Dissertação (Mestrado em Estatística e Experimentação Agropecuária). Lavras: UFLA. 63p. 2013.

BEZERRA, J. E. F.; LEDERMAN, L. E.; SILVA JÚNIOR, J. F. S.; ALVES, M. A. Comportamento da pitangueira (Eugenia uniflora L.) sob irrigação na região do Vale do Rio Moxotó, Pernambuco. Revista Brasileira de Fruticultura, v. 26: 177-179. 2004. 10.1590/S0100-29452004000100047

BOESSO, F. F.; BRUNELLI, L. T.; IMAIZUMI, V. M.; VENTURINI FILHO, W. G. Caracterização físico-química, energética e sensorial de refresco adoçado de jabuticaba. Energia na Agricultura, 30: 429-436. 2015. $\underline{10.17224 / \text { EnergAgric.2015v30n4p429-436 }}$

BORGES, K. C. F.; SANTANA, D. G.; MELO, B.; DOS SANTOS, C. M. Rendimento de polpa e morfometria de frutos e sementes de pitangueira-do-cerrado. Revista Brasileira de Fruticultura, 32: 471-478. 2010. 10.1590/S0100$\underline{29452010005000042}$

BRAGA, L. A. C.; PENHA, F. B.; SOUZA, L. F. A.; BRAGA, A. C. C.; RODRIGUES, E. C. N; BEZERRA, T. S. Sensory profile and physical-chemical evaluation of mixed nectar of Pitaya and Passion fruit. Brazilian Journal of Development, 6: 38970-38987. 2020. 10.1590/S0103-90162004000600007
CARVALHO, J. E. U.; NAZARÉ, R. F. R.; NASCIMENTO, W. M. O. Características físicas e físico-químicas de um tipo de bacuri (Platonia insignis Mart.) com rendimento industrial superior. Revista Brasileira de Fruticultura, 25: 326-328. 2003. $\underline{10.1590 / \mathrm{S} 0100-29452003000200036}$

CARVALHO, A. R. J.; GOMES, G. A.; FERREIRA, R. O.; CARVALHO, M. G. Constituintes químicos e atividade antioxidante de folhas e galhos de Eugenia copacabanensis Kiaersk (Myrtaceae). Química Nova, 37: 477-482. 2014. 10.5935/0100-4042.20140079

COHEN, K. O.; SANO, S. M.; SILVA, J. C. S.; MELO, J. T. Avaliação das características físicas e físico-químicas dos frutos de Ariticum mocedentes de Cabeceiras, Embrapa Cerrado. 2020.

DANNER, M. A.; CITADIN, I.; SASSO, S. A. Z.; SACHET, M. R.; AMBRÓSIO, R. Fenologia da Floração e Frutificação de Mirtáceas Nativas da Floresta com Araucária. Revista Brasileira de Fruticultura, 32: 291-295. 2010. 10.1590/S010029452010005000008 .

DIAS, A. B.; CARVALHO, M. A. P.; DANTAS, A. C. V. L.; FONSECA, V. J. A. Variabilidade e caracterização de frutos de pitangueiras em municípios baianos. Revista Brasileira de Fruticultura, 33: 1169-1177. 2011. 10.1590/S0100$\underline{29452011000400015}$

GUERRA, C. C. Sistema para Elaboração de Suco de Uva Integral em Pequenos Volumes: Suquificador Integral. Empresa Brasileira de Pesquisa Agropecuária, Embrapa Uva e Vinho, Bento Gonçalves - RS. 2016.

GULARTE, M. A. Manual de análise sensorial de alimentos. Editora da Universidade Federal de Pelotas, 106p. 2009.

IBGE. Instituto Brasileiro de Geografia e Estatística. Cidades: Três Passos [website]. Disponível em: <http://cidades.ibge.gov.br/painel/painel.php?codmun=432190> . Acesso em: 10 abril de 2020.

LORENZI, H.; LACERDA, M. Frutas brasileiras e exóticas cultivadas (de consumo in natura). Instituto Plantarum de Estudos da Flora, São Paulo. 672 p. 2006.

MOURA, A. A. C.; AROUCHA, E. M. M.; GÓIS, V. A.; LIETE, R. H. L FERREIRA, R. M. A.; SILVA, M. C. P. Iogurtes com polpa de noni e acerola: avaliação físico-química, atividade antioxidante e perfil sensorial. Boletim CEPPA, 34: 110. $2016.10 .5380 /$ cep.v34i2.53181

REIS, L. C. R. BERNARDI, J. R.; SILVA, A. C. P.; FACCO, E. M. P. Análise da composição nutricional e estabilidade de compostos fenólicos e antocianinas totais do guabijú (Myrcianthes punges). Brazilian Journal of Food Research, 7: 89-104. 2016. 10.3895/rebrapa.v7n1.3517

RODRIGUES, M. A.; GUERRA, D.; SEHN, T. T.; BOHRER, R. E. G.; SILVA, D. M. Caracterização biométrica de 
Guabijuzeiros (Myrcianthes pungens (O. Berg) d. Legrand). Revista Eletrônica Cientifica da UERGS, 6: 83-91. 2020. $\underline{10.21674 / 2448-0479.61 .83-91}$

SERAGLIO, S. K. T.; SCHULZ, M., NEHRING, P.; DELLA BETTA, F.; VALESE, A. C.; DAGUER, H.; GONZAGA, L.V., FETT, R., COSTA, A. C. O. Nutritional and bioactive potential of Myrtaceae fruits during ripening. Food Chem, 239: 649-656, 2018. 10.1016/j.foodchem.2017.06.118

SILVA, J. A. A.; GRIZOTTO, R. K.; MIGUEL, F. B.; BÁRBARO, I. M. Caracterização físico-química de frutos de clones de doviális (Dovyalis abyssinicawarb). Revista Brasileira de Fruticultura, 33: 466-472. 2011. 10.1590/S0100$\underline{29452011000500062}$

SOBUCKI, L.; BETEMPS, D. L.; RAMOS, L. F.; LEDUR, C. L.; ROHRIG, B. Caracterização físico-química de diferente espécie de mirtáceas na cidade de Cerro Largo RS. Cadernos de Agroecologia, 10: 1-5. 2016.

SOUZA, L. S.; AVRELlA, E. D.; CAMPOS, S. S.; FIOR, C. S.; SCHWARZ, S. F. Clonagem de espécime adulto de Myrcianthes pungens (Berg) Legrand através da estaquia. Iheringia, Série Botânica, 73: 336-341. 2018. 10.21826/2446$\underline{8231201873310}$

R CORE TEAM R. 2020. A language and environment for statistical computing. R Foundation for Statistical Computing, Vienna, Austria.

VEIT, P. A.; SCHWARZ, S. F.; GUERRA, D. Monitoring the phenology of Myrcianthes pungens (O. Berg) d. Legrand in the state of Rio Grande do Sul - Brazil. Revista Brasileira de Fruticultura, 41: 1-4. 2019. 10.1590/0100-29452019164

VERGARA, L. P.; RODRIGUES, R. S.; ZAMBIASI, R. C.; FRANZON, R. C.; CHIM, J. F. Sensorial profile of conventional chewable bullets and reduced caloric value formulated with yellow Araçá Pulp. Brazilian Journal of Development, $\quad 6$ : 2020. $\underline{10.1016 / \text { j.foodqual.2008.09.001 }}$

ZERBIELLI, L.; NIENOW, A. A.; DALACORTE, L.; JACOBS, R.; DARONCH, T. Diversidade físico-química dos frutos de jabuticabeiras em um sítio de ocorrência natural. Revista Brasileira de Fruticultura, 38: 107-116, 2016. $\underline{10.1590 / 0100-2945-267 / 14}$. 